\title{
Dielectric Properties of Modified Lead Zirconate Titanate
}

\author{
R.K. Sharma ${ }^{* 1}$ - Gunjan Purohit ${ }^{1}$ D. K. Tyagi ${ }^{2}$ \\ ${ }^{1}$ Department of Physics, D.A.V. Post Graduate College, Dehradun, Uttarakhand-248001, India \\ ${ }^{2}$ Department of Chemistry, D. A. V. Post Graduate College, Dehradun, Uttarakhand-248001, India
}

*Corresponding Author Email id: davrks@gmail.com

Received: 2.8.2021; Revised: 18.11.2021; Accepted: 19.11.2021

(C)Society for Himalayan Action Research and Development

\begin{abstract}
In this paper, the dielectric properties of modified i. e. alkali doped lead zirconate titanate $\mathrm{Pb}\left(\mathrm{Zr}_{1-}\right.$ $\left.{ }_{\mathrm{x}} \mathrm{T}_{\mathrm{ix}}\right) \mathrm{O}_{3}$ has been studied. The composition $\mathrm{Pb}_{1-\mathrm{x}}\left(\mathrm{La}_{1-\mathrm{z}} \mathrm{D}_{\mathrm{z}}\right)_{\mathrm{x}}\left(\mathrm{Zr}_{\mathrm{y}} \mathrm{Ti}_{1-\mathrm{y}}\right)_{1-\mathrm{x} / 4} \mathrm{O}_{3}$; where $\mathrm{x}=0.10, \mathrm{y}=0.70, \mathrm{z}=0.0$, $0.1,0.2$, and $0.3, \mathrm{D}=\mathrm{Na}, \mathrm{K}$ and $\mathrm{Li}$ has been prepared by conventional mixed oxide method at a temperature of $1100^{\circ} \mathrm{C}$. Dielectric constant and dielectric loss measurement of the sintered pellets has been measured. Dielectric measurement shows that the dielectric constant decreases with an increase in temperature. The results show that the dielectric loss is very small and decreases with frequency above $300^{\circ} \mathrm{C}$. The increase in the dielectric constant observed at high temperatures and low frequencies is explained in the paraelectric state.
\end{abstract}

Keywords: Dielectric properties, PZT, Dielectric constant, Dielectric loss

\section{Introduction}

Lead zirconate titanate (PZT) in polycrystalline form is one of the most well-known ferroelectric materials (Jaffe et al. 1971; Shkuratov, 2019) because of its technological importance and versatile properties, especially in the morphotropic phase boundary (MPB) region that occurs at nearly equal concentrations of $\mathrm{Ti}^{4+}$ and $\mathrm{Zr}^{4+}$ (Gonçalves et al., 2016).The dielectric constants and piezoelectric coefficients in this region show an anomalously sharp maximum during the transition from Ti-rich tetragonal to $\mathrm{Zr}$-rich rhombohedral phases (Noheda, 2002).Compositionally modified lead zirconate titanate (doped-PZT) ceramics are widely used in dielectric, and piezoelectric applications such as high permittivity capacitors, piezoelectric elements, sensors, pyroelectric detectors and ferroelectric memories etc. (Xu, 1991; Izyumskaya et al. 2007; Thakur et al. 2007; Silva Neto et al. 2012). The physical properties of the PZT ceramics depend mainly on the temperature and the time of densification. The initial chemical composition may be disturbed as the lead evaporates during the densification process. PZT has a high dielectric constant, ferroelectric, piezoelectric, and pyroelectric properties. Depending on the orientation and doping, the dielectric constant of PZT can range from 300 to 3850 (Sundar et al. 2018).

Dielectric analysis is an important feature that can be used to gain knowledge about the electrical properties of a material medium as a function of temperature and frequency. The ability of storing electric charges by the material and capability of transferring 
the electric charge can be assessed by the dielectric analysis. Much work has been done on the dielectric and electrical properties of lead zirconate titanate in various compositions (Balusamy et al. 2015; Oliveira et al. 2014; Sharma et al. 2020; Unruan et al. 2009). The dielectric, piezoelectric and pyroelectric properties of PZT can be modified by adding dopants (Kour et al. 2016; Zachariasz and Bochenek, 2009). Sahoo and Panda (2013) have studied the effect of $\mathrm{La}^{3+}$ and $\mathrm{Nd}^{3+}$ dopants on piezoelectric, dielectric and ferroelectric properties of PZTs. They showed that $\mathrm{La}^{3+}$ dopant is more effective than $\mathrm{Nd}^{3+}$, which contains a mixture of $\mathrm{La}^{3+}$ and $\mathrm{Nd}^{3+}$ dopants. Donor dopants such as $\mathrm{La}^{3+}$ and $\mathrm{Nd}^{3+}$ produce soft PZT, which further facilitate the domain wall motion and enhances the electronic properties of PZT compared to undoped PZT. Mirzaei et al. (2016) have studied the effect of $\mathrm{Nb}$ doping on the sintering and dielectric properties of PZT ceramics. They found that Nb-doped PZT ceramics had higher dielectric permittivity $\left(\varepsilon_{r} \sim 17960\right)$ with a lower Curie temperature $\left(\sim 358{ }^{\circ} \mathrm{C}\right)$ relative to PZT $\left(\varepsilon_{r}=16000\right.$ at $\sim 363{ }^{\circ} \mathrm{C}$ ). Sun et al. (2015) have studied the effect of $\mathrm{Mn} / \mathrm{Nb}$ doping on dielectric and ferroelectric properties of PZT thin films. For $\mathrm{Mn} / \mathrm{Nb}$-doped lead zirconate titanate thin films, their results showed that the ferroelectric and dielectric properties were deteriorate at low Mn concentrations and decrease as the increase of $\mathrm{Nb}$ doping level. Xu et al. (2017) have investigated the effect of La doping on the dielectric properties of PZT thin films. They obtained the maximum dielectric constant
$(1502.59$ at $100 \mathrm{~Hz})$ in a 2at. -\% La-doped film, which was a $53.9 \%$ increase compared to the undoped film.

In the present work, we have investigated the synthesis and dielectric properties of alkali ( $\mathrm{Na}, \mathrm{K}$ and $\mathrm{Li}$ ) doped lead zirconate titanate systems. The polycrystalline material synthesized in the present investigation is $\mathrm{Pb}_{1-\mathrm{x}}\left(\mathrm{La}_{1-}\right.$ $\left.{ }_{2} \mathrm{D}_{\mathrm{z}}\right)_{\mathrm{x}}\left(\mathrm{Zr}_{\mathrm{y}} \mathrm{Ti}_{1-\mathrm{y}}\right)_{1-\mathrm{x} / 4} \mathrm{O}_{3}$; where $\mathrm{x}=0.10, \mathrm{y}=$ $0.70, \mathrm{z}=0.0,0.1,0.2$, and $0.3, \mathrm{D}=\mathrm{Na}, \mathrm{K}$ and $\mathrm{Li}$.

\section{Experimental Procedure}

Polycrystalline ceramic of $\mathrm{Pb}_{1-\mathrm{x}}\left(\mathrm{La}_{1-}\right.$ $\left.{ }_{2} \mathrm{D}_{\mathrm{z}}\right)_{\mathrm{x}}\left(\mathrm{Zr}_{\mathrm{y}} \mathrm{Ti}_{1-\mathrm{y}}\right)_{1-\mathrm{x} / 4} \mathrm{O}_{3}$ was prepared using the conventional mixed oxide method. High purity analytical grade raw materials were used to prepare the ferroelectric PZT ceramics. Zirconium and titanium citrates with known concentrations were used to prepare PZT powders in the ratio $\mathrm{Zr} / \mathrm{Ti}$ of $70 / 30$. Stoichiometric amounts of metal oxides and metal carbonates were weighed and mixed according the molecular formula. The raw materials are mixed in acetone medium for 50 to 60 minutes with the help of agate mortar and pestle for homogeneous mixing of the mixture. Since calcination is necessary for the completion of solid-state reaction, therefore, the composite materials were calcined at $1000^{\circ} \mathrm{C}$ for 2 hours. The pellets were formed by the powder with the help of uni-axial hydraulic press and die punch. To make the pellets more compact with reduce porosity, the pellets were sintered at $1100^{\circ} \mathrm{C}$ for 4 to 6 hours. The dielectric study (dielectric constant and dielectric loss measurement) of 
sintered pellets were performed by LCR controller (Hioki 3522-50).

\section{Results and Discussion}

The variation of dielectric constant $(\varepsilon)$ and dielectric loss $(\tan \delta)$ with the frequency of all samples at room temperature has been measured in the frequency range of $10^{2} \mathrm{~Hz}$ to $10^{5} \mathrm{~Hz}$. It is found that dielectric constant and dielectric loss decrease with increase in frequency. Generally, both of these parameters decrease with increase in frequency and show a characteristic feature of a dielectrics. The mechanism of variation in dielectric constant of the ceramics with frequency can be explained in terms of four types of polarization that contribute to the dielectric constant of material. These are: electronic, ionic, orientation and space charge polarization. At very low frequency (less than $10 \mathrm{kHz}$ ), all the contributions may be active. From the nature of the variation of $\varepsilon$ with frequency, it is possible to find out which contributions are predominately present in the compound in a particular frequency range. Only the contribution of polar regions to the total dielectric constant in the region of frequency under investigation undergo dispersion. All these spontaneously polarizable regions make a contribution to the dielectric constant only at temperature below the transition point. Increase in the space charge polarization of a solid increases the value of $\varepsilon$ and $\tan \delta$. This polarization arises due to defects and impurities present either in the bulk or at the surface of the crystal or both. Due to large polarization of defects in the crystals, the space charge polarization increases and thereby $\varepsilon$ and $\tan \delta$ of the crystal become high. The dipolar orientation effect can sometimes be exhibited by some materials even up to $10^{10} \mathrm{~Hz}$. The ionic and electronic polarizations always exist above $10^{13}$ Hz. Only pure electronic polarization exists above $10^{15} \mathrm{~Hz}$ as a result of which the dielectric constant of the material falls down to a very low value.

Pressed ceramic samples generally contain voids, grain boundaries and other defects. The presence of voids decreases the dielectric constants. However, if the density of the sample approaches the single crystal value, then this effect is practically eliminated. The number of polarizable entities will be enhanced due to increase in density or packing fraction and they exhibit larger $\varepsilon$ values. The dielectric loss in pellet samples is mostly due to the scattering mechanism. The scattering cross section depends upon grain size, inter grain space and grain boundaries. Therefore, the loss factor decreases smoothly with increase of frequency because of high packing fraction of pellet. The smaller grain size also affects the scattering and increases the scattering amplitude. Furthermore, the space charge may arise from the charges present at the surface of the crystallites.

The magnitude of the dielectric constant depends on the doping and the frequency measured. The frequency dependence of the dielectric constant shows strong 
dispersion over the low frequency range. This phenomenon has been attributed to the low frequency space charge accumulation effect. Strong dispersion in the dielectric constant is a common feature of ferroelectrics associated with non-negligible ionic conductivity and is called low frequency dielectric dispersion (Prasad et al. 1993).The region around the dielectric peak appears to be broad due to compositional fluctuations, which is one of the most important features of a disordered perovskite structure with diffuse phase transition (Goel et al. 2004).In $\mathrm{ABO}_{3}$ type compounds, compositional fluctuations can develop at the A site occupied by $\mathrm{Pb}^{2+}$, alkali elements $\left(\mathrm{Li}^{+}, \mathrm{Na}^{+}, \mathrm{K}^{+}\right)$or $\mathrm{La}^{3+}$ or at the $\mathrm{B}$ site occupied by $\mathrm{Ti}^{4+}$, $\mathrm{Zr}^{4+}$ or $\mathrm{La}^{3+}$.

Figures 1 to 5 show the variation of dielectric constant $(\varepsilon)$ and dielectric loss

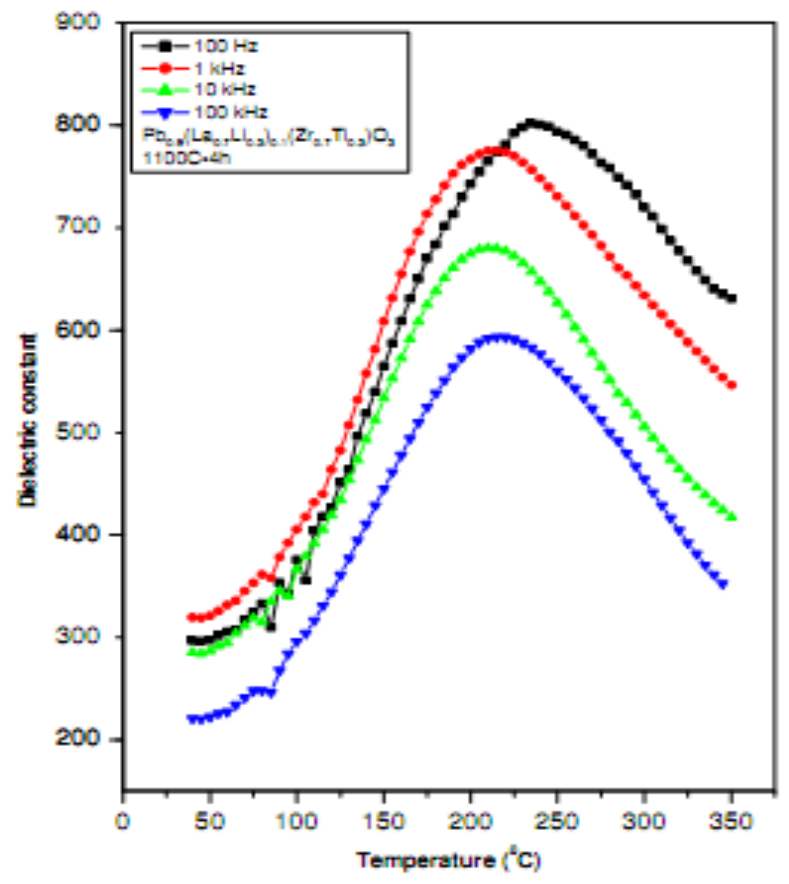

(a)

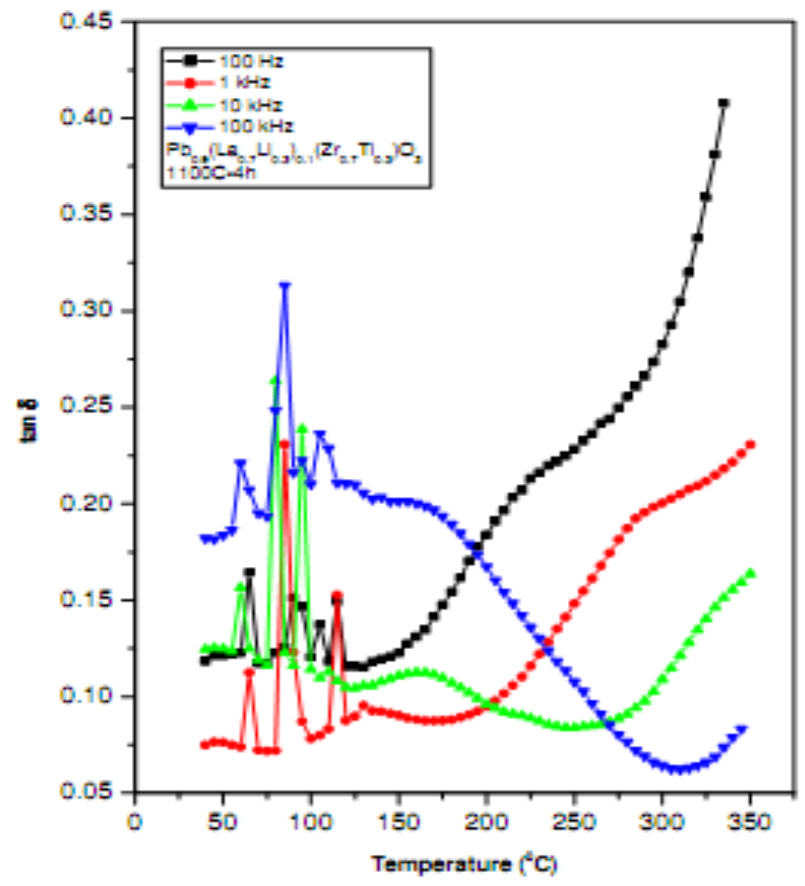

(b)

Figure 1. Variation of dielectric constant (a) and dielectric loss (b) with temperature at different frequency for $\mathrm{Pb}_{0.9}\left(\mathrm{La}_{0.7} \mathrm{Li}_{0.3}\right)_{0.1}\left(\mathrm{Zr}_{0.7} \mathrm{Ti}_{0.3}\right) \mathrm{O}_{3}\left(1100^{\circ} \mathrm{C}, 6\right.$ hours $)$. 


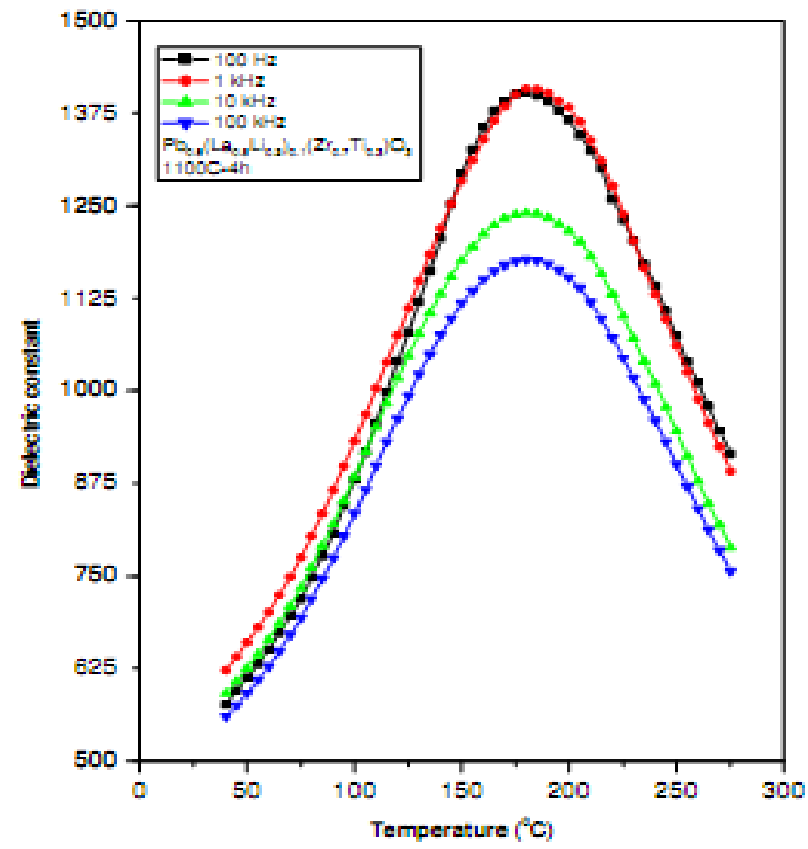

(a)

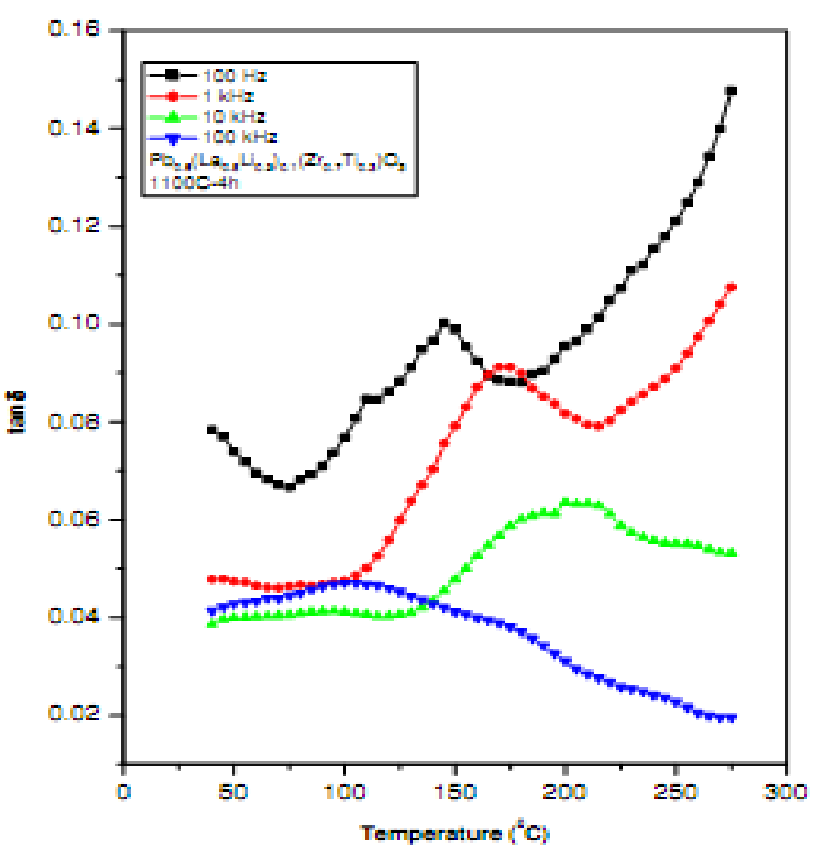

(b)

Figure 2. Variation of dielectric constant (a) and dielectric loss (b) with temperature at different frequency for $\mathrm{Pb}_{0.9}\left(\mathrm{La}_{0.8} \mathrm{Li}_{0.2}\right)_{0.1}\left(\mathrm{Zr}_{0.7} \mathrm{Ti}_{0.3}\right) \mathrm{O}_{3}\left(1100^{\circ} \mathrm{C}, 6\right.$ hours $)$.

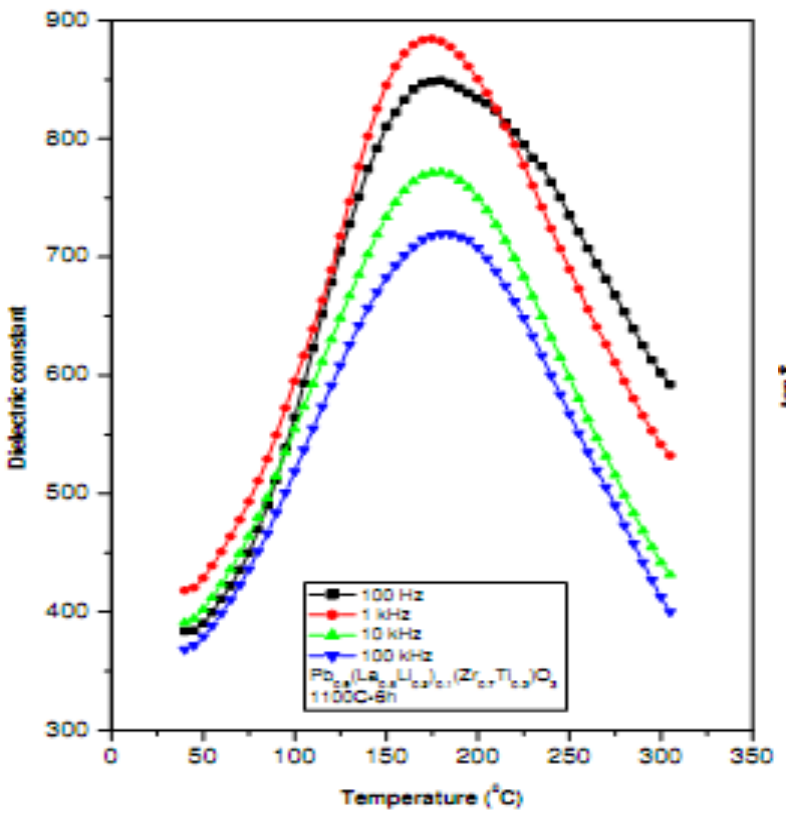

(a)

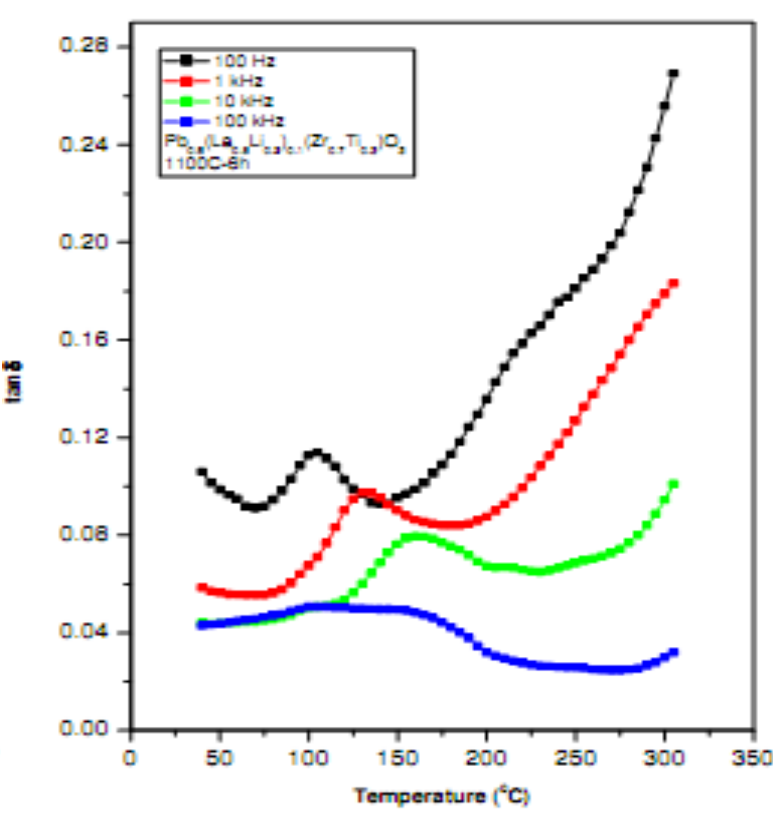

(b)

Figure 3. Variation of dielectric constant (a) and dielectric loss (b) with temperature at different frequency for $\mathrm{Pb}_{0.9}\left(\mathrm{La}_{0.8} \mathrm{Li}_{0.2}\right)_{0.1}\left(\mathrm{Zr}_{0.7} \mathrm{Ti}_{0.3}\right) \mathrm{O}_{3}\left(1100^{\circ} \mathrm{C}, 4\right.$ hours $)$

The dielectric study indicates that alkali doped PZT ceramics undergo a diffuse type of phase transition. The expansion of the dielectric peak and the decrease in the 


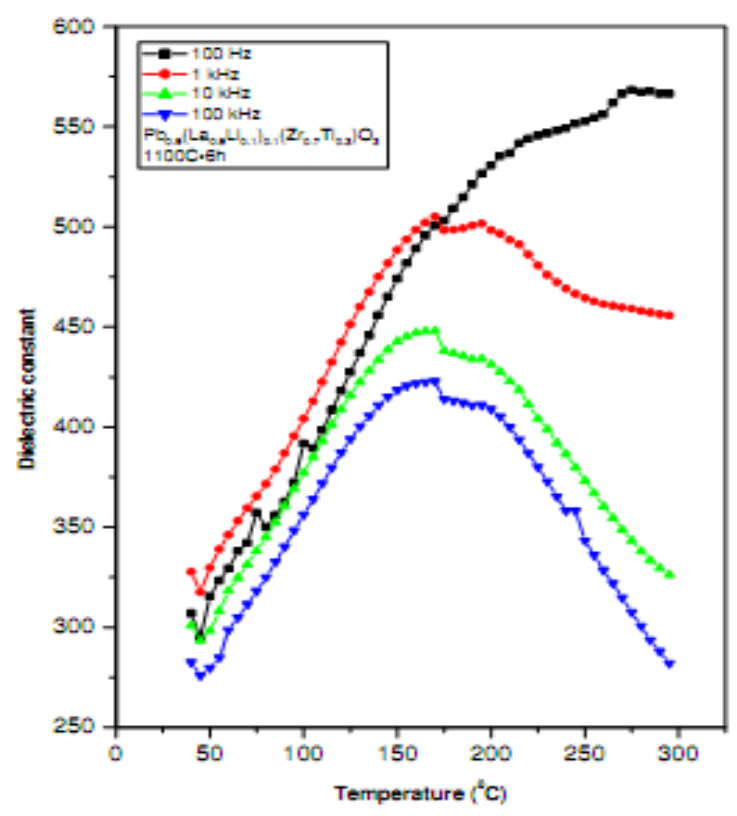

(a)

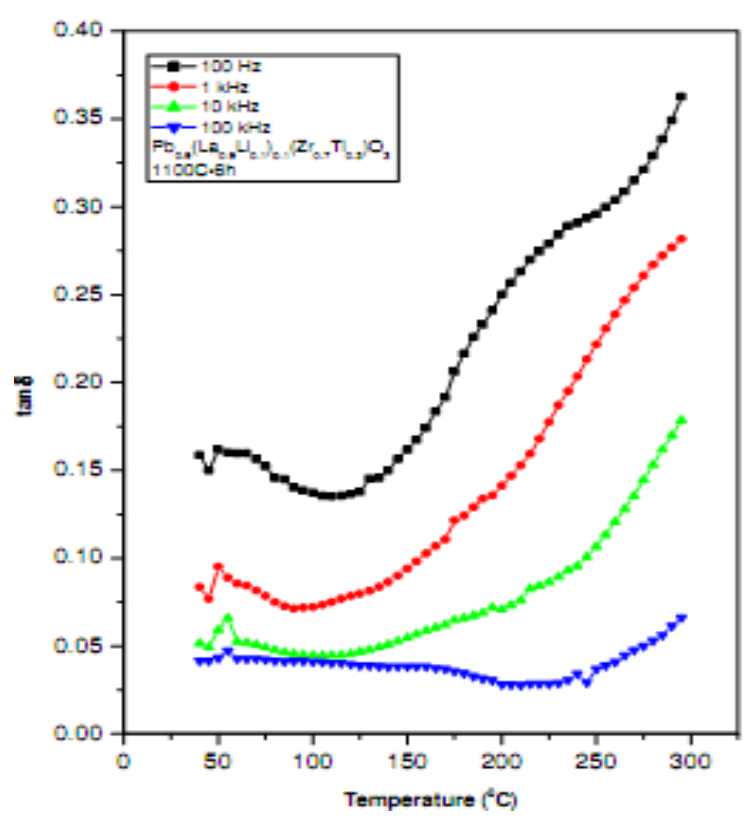

(b)

Figure 5. Variation of dielectric constant (a) and dielectric loss (b) with temperature at different frequency for $\mathrm{Pb}_{0.9}\left(\mathrm{La}_{0.9} \mathrm{Li}_{0.1}\right)_{0.1}\left(\mathrm{Zr}_{0.7} \mathrm{Ti}_{0.3}\right) \mathrm{O}_{3}\left(1100^{\circ} \mathrm{C}, 4\right.$ hours $)$.

maximum value of dielectric constant correspond to a decrease in the grain size as the concentration of dopants increases. High dielectric constant and low dielectric loss have been observed for all compositions. This study may be useful for many applications.

\section{References}

Balusamy R, Kumaravel $\mathrm{P}$ and Renganathan N G (2015). Dielectric and electrical properties of lead zirconate titanate. Der Pharma Chemica, 7: 175-185.

Goel P, Yadav K L and JamesA R (2004). Double doping effect on the structural and dielectric properties of PZT ceramics. J Phys D :Appl Phys. 37 :3174-3170.

Gonçalves M D, Souza F L, Longo E, Leite E R and Camargo E R (2016). Dielectric characterization of microwave sintered lead zirconate titanate ceramics. Ceramics International 42: 1442314430.
Izyumskaya N, Alivov Y-I, Cho S-J, Morkoç H, Lee $\mathrm{H}$ and Kang $\mathrm{Y}-\mathrm{S}$ (2007). Processing, Structure, Properties, and Applications of PZT Thin Films. Critical Reviews in Solid State and Materials Sciences 32: 111202.

Jaffe B, Cook W R and Jaffe B 1971 Piezoelectric ceramics, USA: Academic Press, New York.

Kour P, Pradhan S.K, Kumar P, Sinha S K and Kar M (2016). Enhanced ferroelectric and piezoelectric properties in La-modified PZT ceramics. Appl. Phys. A 122:591.

Mirzaei A, Bonyani $M$ and Torkian $S$ (2016). Effect of $\mathrm{Nb}$ doping on sintering and dielectric properties of PZT ceramics. Processing and Application of Ceramics 10: 175-182.

Noheda B (2002). Structure and highpiezoelectricity in lead oxide solid solutions. Curr. Opin. Solid State Mater. Sci. 6: 27-34.

Oliveira C A, Longo E, Varela J A and Zaghete M A (2014). Synthesis and characterization of lead zirconate 
titanate (PZT) obtained by two chemical methods. Ceramics International40:1717-1722.

Prasad K, Sati R, Choudhary S N, Choudhary R N P and Yadav K L (1993),

$(\mathrm{Pb}, \mathrm{Ca})\left[\left(\mathrm{Mn}_{0.5} \mathrm{~W}_{0.5}\right), \mathrm{Ti}\right] \mathrm{O}_{3}$ ceramics:

$\mathrm{X}$-ray and dielectric studies. J. Mater. Sci. Lett. 12: 758-759.

Sahoo B and Panda P K (2013). Effect of lanthanum, neodymium on piezoelectric, dielectric and ferroelectric properties of PZT. Journal of Advanced Ceramics 2: 3741.

Sharma R K, Tyagi D K and Purohit G (2020). Dielectric properties of lead zirconate titanate. Applied Innovative Research 2: 204-207.

Shkuratov S I (2019). Explosive Ferroelectric Generators: From Physical Principles to Engineering, Singapore: World Scientific, Pp. 2544.

Silva Neto L P, Rossi J O and Silva A R (2012). Applications of PZT Dielectric Ceramics in High-Energy Storage Systems. Materials Science Forum 727-728: 505-510.

Sun H, Zhang Y, Liu X, Shanshan G, Liu $\mathrm{Y}$ and Chen W. (2015). The effect of $\mathrm{Mn} / \mathrm{Nb}$ doping on dielectric and ferroelectric properties of PZT thin films prepared by sol-gel process. $J$. Sol-Gel Science and Technology 74: 378-386.

Sundar U, Banerjee S and Cook-Chennault K A (2018). Piezoelectric and dielectric properties of PZT epoxy composite thick films. Academ. J. Polym. Sci.: 95-109.
Thakur O P, Singh J P, Prakash C and Kishan P (2007). Modified Leadzirconate-titanate for pyroelectric sensors. Defense Science Journal 57: 233-239.

Unruan M, Prasartketrakarn A, Ngamjarurojana A, Laosiritaworn Y, Ananta Sand Yimnirun R (2009).Dielectric and ferroelectric properties of lead zirconate titanatelead nickel niobate ceramics under compressive stress. J. Appl. Phys. 105 :084111.

$\mathrm{Xu}$ W, Li Q, Yin Z, Wang $\mathrm{X}$ and Zou H (2017). Effect of La doping on crystalline orientation, microstructure and dielectric properties of PZT thin films. Materials Testing 59:885-889.

$\mathrm{Xu}$ Y (1991). Ferroelectric Materials and Their Applications, North Holland, Amsterdam.

Zachariasz R and Bochenek D (2009). Properties of the PZT type ceramics admixed with barium and niobium.Archives of Metallurgy and Materials 54: 895-902. 